\title{
Adaptive Mesh Refinement for Global Magnetohydrodynamic Simulation
}

\author{
Tamas I. Gombosi, Darren L. De Zeeuw, Kenneth G. Powell, Aaron J. Ridley, \\ Igor V. Sokolov, Quentin F. Stout, and Gábor Tóth^ \\ Center for Space Environment Modeling, The University of Michigan, Ann Arbor, \\ Michigan, USA
}

\begin{abstract}
The first part of this paper reviews some physics issues representing major computational challenges for global MHD models of the space environment. These issues include: (i) mathematical formulation and discretization of the governing equations that ensure the proper jump conditions and propagation speeds, (ii) regions of relativistic Alfvén speed, (iii) regions dominated by strong intrinsic planetary magnetic field with strong gradients, and (iv) the religiously debated issue of controling the divergence of the magnetic field. The second part of the paper concentrates to modern solution methods that have been developed by the aerodynamics, applied mathematics and DoE communities. Such methods have recently begun to be implemented in space-physics codes, which solve the governing equations for a compressible magnetized plasma. These techniques include high-resolution upwind schemes, block-based solution-adaptive grids and domain decomposition for parallelization. While some of these techniques carry over relatively straightforwardly to space physics, space physics simulations pose some new challenges. We give a brief review of the state-of-the-art in modern space-physics codes. Finally, we describe the space physics MHD code developed at the University of Michigan and its recent coupling to a thermosphere-ionosphere and inner magnetosphere model.
\end{abstract}

\section{Introduction}

Global computational models based on first principles represent a very important component of efforts to understand the intricate processes coupling the Sun to the geospace environment. The hope for such models is that they will eventually fill the gaps left by measurements, extending the spatially and temporarily limited observational database into a self-consistent global understanding of our space environment.

Presently, and in the foreseeable future, magnetohydrodynamic (MHD) models are the only models that can span the enormous distances present in the magnetosphere. However, it should not be forgotten that even generalized MHD equations are only a relatively low-order approximation to more complete physics; they provide only a simplified description of natural phenomena in space plasmas.

^ also at Department of Atomic Physics, Loránd Eötvös University, Budapest, Hungary 


\section{Non-relativistic Magnetohydrodynamics}

The governing equations for an ideal, non-relativistic, compressible plasma may be written in a number of different forms. While the different forms of the MHD equations describe the same physics at the differential equation level, there are important practical differences when one solves discretized forms of the various formulations.

According to the Lax-Wendroff theorem [37] only conservative schemes can be expected to get the correct jump conditions and propagation speed for a discontinuous solution. This fact is much less emphasized in the global magnetosphere simulation literature than the more controversial divergence of $B$ issue. In some test problems the non-conservative discretization of the MHD equations can lead to significant errors, which do not diminish with increased grid resolution.

\subsection{Primitive Variable Form}

In primitive variables, the governing equations of ideal magnetohydrodynamics, which represent a combination of the Euler equations of gasdynamics and the Maxwell equations of electromagnetics, may be written as:

$$
\begin{gathered}
\frac{\partial \rho}{\partial t}+\mathbf{u} \cdot \nabla \rho+\rho \nabla \cdot \mathbf{u}=0 \\
\rho \frac{\partial \mathbf{u}}{\partial t}+\rho \mathbf{u} \cdot \nabla \mathbf{u}+\nabla p-\frac{1}{\mu_{0}} \mathbf{j} \times \mathbf{B}=0 \\
\frac{\partial \mathbf{B}}{\partial t}+\nabla \times \mathbf{E}=0 \\
\frac{\partial p}{\partial t}+\mathbf{u} \cdot \nabla p+\gamma p \nabla \cdot \mathbf{u}=0
\end{gathered}
$$

where $\mu_{0}$ and $\gamma$ represent the magnetic permeability of vacuum and the specific heat ratio of the gas. In addition, the current density, $\mathbf{j}$, and the electric field vector, E, are related to the magnetic field B by Ampère's law and Ohm's law:

$$
\begin{aligned}
& \mathbf{j}=\frac{1}{\mu_{0}} \nabla \times \mathbf{B} \\
& \mathbf{E}=-\mathbf{u} \times \mathbf{B}
\end{aligned}
$$

\subsection{Gasdynamic Conservation Form}

For one popular class of schemes, the equations are written in a form in which the gasdynamic terms are put in divergence form, and the electromagnetic terms in the momentum and energy equations are treated as source terms. This gives:

$$
\begin{gathered}
\frac{\partial \rho}{\partial t}+\nabla \cdot(\rho \mathbf{u})=0 \\
\frac{\partial(\rho \mathbf{u})}{\partial t}+\nabla \cdot(\rho \mathbf{u u}+p \mathbf{I})=\frac{1}{\mu_{0}} \mathbf{j} \times \mathbf{B} \\
\frac{\partial \mathbf{B}}{\partial t}+\nabla \times \mathbf{E}=0 \\
\frac{\partial E_{g d}}{\partial t}+\nabla \cdot\left[\mathbf{u}\left(E_{g d}+p\right)\right]=\frac{1}{\mu_{0}} \mathbf{j} \cdot \mathbf{E}
\end{gathered}
$$


where $\mathbf{I}$ is the identity matrix and $E_{g d}$ is the gasdynamic total energy, given by

$$
E_{g d}=\frac{1}{2} \rho u^{2}+\frac{1}{\gamma-1} p
$$

This formulation is used in several popular magnetosphere codes [2049], as well as in the publicly available Zeus code 63.

\subsection{Fully Conservative Form}

The fully conservative form of the equations is

$$
\frac{\partial \mathbf{U}}{\partial t}+(\nabla \cdot \mathbf{F})^{\mathrm{T}}=0
$$

where $\mathbf{U}$ is the vector of conserved quantities and $\mathbf{F}$ is a flux diad,

$$
\begin{gathered}
\mathbf{U}=\left(\begin{array}{c}
\rho \\
\rho \mathbf{u} \\
B \\
E_{m h d}
\end{array}\right) \\
\mathbf{F}=\left(\begin{array}{c}
\rho \mathbf{u} \\
\rho \mathbf{u} \mathbf{u}+\left(p+\frac{1}{2 \mu_{0}} B^{2}\right) \mathbf{I}-\frac{1}{\mu_{0}} \mathbf{B B} \\
\mathbf{u B}-\mathbf{B u} \\
\mathbf{u}\left(E_{m h d}+p+\frac{1}{2 \mu_{0}} B^{2}\right)-\frac{1}{\mu_{0}}(\mathbf{u} \cdot \mathbf{B}) \mathbf{B}
\end{array}\right)^{\mathrm{T}}
\end{gathered}
$$

where $E_{m h d}$ is the magnetohydrodynamic energy, given by

$$
E_{m h d}=E_{g d}+\frac{1}{2 \mu_{0}} B^{2}
$$

\subsection{Symmetrizable Formulation}

Symmetrizable systems of conservation laws have been studied by Godunov [21] and Harten [30], among others. One property of the symmetrizable form of a system of conservation laws is that an added conservation law

$$
\frac{\partial(\rho s)}{\partial t}+\frac{\partial\left(\rho s u_{x}\right)}{\partial x}+\frac{\partial\left(\rho s u_{y}\right)}{\partial y}+\frac{\partial\left(\rho s u_{z}\right)}{\partial z}=0
$$

for the entropy $s$ can be derived by a linear combintion of the system of equations. For the ideal MHD equations, as for the gasdynamic equations, the entropy is $s=\log \left(p / \rho^{\gamma}\right)$. Another property is that the system is Galilean invariant; all waves in the system propagate at speeds $u \pm c_{w}$ (for MHD, the possible values of $c_{w}$ are the Alfvén, magnetofast and magentoslow speeds). Neither of these properties holds for the fully conservative form of the MHD equations. 
Godunov showed that the fully conservative form of the MHD equations (eq. 5]) is not symmetrizable 21]. The symmetrizable form may be written as

$$
\frac{\partial \mathbf{U}}{\partial t}+(\nabla \cdot \mathbf{F})^{\mathrm{T}}=\mathbf{Q}
$$

where

$$
\mathbf{Q}=-\nabla \cdot \mathbf{B}\left(\begin{array}{c}
0 \\
\frac{1}{\mu_{0}} \mathbf{B} \\
\mathbf{u} \\
\frac{1}{\mu_{0}} \mathbf{u} \cdot \mathbf{B}
\end{array}\right)
$$

Vinokur separately showed that eq. (8) can be derived starting from the primitive form, if no stipulation is made about $\nabla \cdot \mathbf{B}$ in the derivation. Powell showed that this symmetrizable form can be used to derive a Roe-type approximate Riemann solver for solving the MHD equations in multiple dimensions [45].

The MHD eigensystem arising from eq. (5) or eq. (8) leads to eight eigenvalue/eigenvector pairs. The eigenvalues and associated eigenvectors correspond to an entropy wave, two Alfvén waves, two magnetofast waves, two magnetoslow waves, and an eighth eigenvalue/eigenvector pair that depends on which form of the equations is being solved. This last wave (which describes the jump in the normal component of the magnetic field at discontinuities) has a zero eigenvalue in the fully conservative case, and an eigenvalue equal to the normal component of the velocity, $u_{n}$, in the symmetrizable case. The expressions for the eigenvectors, and the scaling of the eigenvectors, are more intricate than in gasdynamics [59].

We note that while eq.(5) is fully conservative, the symmetrizable formulation (given by eq. 8) is formally not fully conservative. Terms of order $\nabla \cdot \mathbf{B}$ are added to what would otherwise be a divergence form. The danger of this is that shock jump conditions may not be correctly met, unless the added terms are small, and/or they alternate in sign in such a way that the errors are local, and in a global sense cancel in some way with neighboring terms. This downside, however, has to be weighed against the alternative; a system (i.e., the one without the source term) that, while conservative, is not Gallilean invariant, has a zero eigenvalue in the Jacobian matrix, and is not symmetrizable.

\section{Semi-relativistic Plasmas}

While the solar-wind speed remains non-relativistic in the solar system, the intrinsic magnetic fields of several planets in the solar system are high enough, and the density of the solar wind low enough, that the Alfvén speed,

$$
V_{\mathrm{A}}=\sqrt{\frac{B^{2}}{\mu_{0} \rho}}
$$

can reach appreciable fractions of the speed of light. In the case of Jupiter, the Alfvén speed in the vicinity of the poles is of order ten! Even Earth has a strong 
enough intrinsic magnetic field that the Alfvén speed reaches twice the speed of light in Earth's near-auroral regions.

\subsection{Limiting the Alfvén Speed}

For these regions, solving the non-relativistic ideal MHD equations does not make sense. Having waves in the system propagating faster than the speed of light, besides being non-physical, causes a number of numerical difficulties. However, solving the fully relativistic MHD equations is overkill. What is called for is a semi-relativistic form of the equations, in which the flow speed and acoustic speed are non-relativistic, but the Alfvén speed can be relativistic. A derivation of these semi-relativistic equations from the fully relativistic equations is given in 25]; the final result is presented here.

The semi-relativistic ideal MHD equations are of the form

$$
\frac{\partial \mathbf{U}_{s r}}{\partial t}+\left(\nabla \cdot \mathbf{F}_{s r}\right)^{\mathrm{T}}=0
$$

where the state vector, $\mathbf{U}_{s r}$, and the flux diad, $\mathbf{F}_{s r}$, are

$$
\begin{gathered}
\mathbf{U}_{s r}=\left(\begin{array}{c}
\rho \\
\rho \mathbf{u}+\frac{1}{c^{2}} \mathbf{S}_{\mathrm{A}} \\
\mathbf{B} \\
\frac{1}{2} \rho u^{2}+\frac{1}{\gamma-1} p+e_{\mathrm{A}}
\end{array}\right) \\
\mathbf{F}_{s r}=\left(\begin{array}{c}
\rho \mathbf{u} \\
\rho \mathbf{u} \mathbf{u}+\mathbf{I}_{\mathrm{A}} \\
\mathbf{u} \mathbf{B}-\mathbf{B} \mathbf{u} \\
\left(\frac{1}{2} \rho u^{2}+\frac{\gamma}{\gamma-1} p\right) \mathbf{u}+\mathbf{S}_{\mathrm{A}}
\end{array}\right)^{\mathrm{T}}
\end{gathered}
$$

In the above,

$$
\begin{gathered}
\mathbf{S}_{\mathrm{A}}=\frac{1}{\mu_{0}}(\mathbf{E} \times \mathbf{B}) \\
e_{\mathrm{A}}=\frac{1}{2 \mu_{0}}\left(B^{2}+\frac{1}{c^{2}} E^{2}\right) \\
\mathbf{P}_{\mathrm{A}}=e_{\mathrm{A}} \mathbf{I}-\frac{1}{\mu_{0}} \mathbf{B} \mathbf{B}-\frac{1}{\mu_{0} c^{2}} \mathbf{E} \mathbf{E}
\end{gathered}
$$

are the Poynting vector, the electromagnetic energy density, and the electromagnetic pressure tensor, respectively. The electric field $\mathbf{E}$ is related to the magnetic field B by Ohm's law, (eq. 2b).

\subsection{Lowering the Speed of Light}

This new system of equations has wave speeds that are limited by the speed of light; for strong magnetic fields, the modified Alfvén speed (and the modified magnetofast speed) asymptote to $c$. The modified magnetoslow speed asymptotes 
to $a$, the acoustic speed. This property offers the possibility of a rather tricky convergence-acceleration technique for explicit time-stepping schemes, first suggested by Boris [11; the wave speeds can be lowered, and the stable time-step thereby raised, by artificially lowering the value taken for the speed of light. This method is known as the "Boris correction."

The equations in Sect. 3.1 are valid in physical situations in which $V_{\mathrm{A}}>c$. A slight modification yields a set of equations, the steady-state solutions of which are independent of the value taken for the speed of light. Defining the true value of the speed of light to be $c_{0}$, to distinguish it from the artificially lowered speed of light, $c$, the equations are:

$$
\frac{\partial \mathbf{U}_{s r}}{\partial t}+\left(\nabla \cdot \mathbf{F}_{s r}\right)^{\mathrm{T}}=\mathbf{Q}_{c_{0}}
$$

where the state vector, $\mathbf{U}_{s r}$, and the flux diad, $\mathbf{F}_{s r}$, are as defined above, and the new source term in the momentum equation is

$$
\mathbf{Q}_{c_{0}}=\frac{1}{\mu_{0}}\left(\frac{1}{c_{0}^{2}}-\frac{1}{c^{2}}\right) \mathbf{E} \nabla \cdot \mathbf{E}
$$

An implementation of the semi-relativistic equations has been made in the BATSRUS code developed at the University of Michigan [4625].

\section{Splitting the Magnetic Field}

For problems in which strong externally imposed magnetic fields are present, accuracy can be increased by solving for the deviation of the magnetic field from this prescribed component. For instance, in magnetosphere-type simulations a strong dipole-like magnetic field dominates the solution near the body. Solving for the deviation $\mathbf{B}_{1}$ from the embedded field $\mathbf{B}_{0}$ is inherently more accurate than solving for the full magnetic field vector $\mathbf{B}=\mathbf{B}_{0}+\mathbf{B}_{1}$. This approach was first suggested by Ogino and Walker [42], applied to Godunov-type schemes by Tanaka 64] and later employed by our group [46]. Recently we generalized it to semi-relativistic MHD [25].

The full magnetic field vector $\mathbf{B}$ can be written as

$$
\mathbf{B}=\mathbf{B}_{0}+\mathbf{B}_{1}
$$

where $\mathbf{B}_{0}$ is given analytically and thus $\nabla \cdot \mathbf{B}_{0}=0$, while $\mathbf{B}_{1}$ is calculated by the numerical scheme. Note that $\mathbf{B}_{1}$ is not necessarily small relative to $\mathbf{B}_{0}$. We also introduce the non-relativistic current density $\mathbf{j}_{0}=\left(1 / \mu_{0}\right) \nabla \times \mathbf{B}_{0}$. The splitting is most important when the equations are solved in a (near) conservation form, since the total energy density can be completely dominated by the magnetic energy $\mathbf{B}_{0}^{2} /\left(2 \mu_{0}\right)$. When the pressure is calculated from the total energy density, it can easily become negative, as we take differences of two huge numbers to obtain a small one. This problem can be mitigated by rewriting the energy equation in terms of the modified total energy density

$$
e_{1}=E_{g d}+\frac{1}{2 \mu_{0}}\left(B_{1}^{2}+\frac{1}{c^{2}} E^{2}\right)
$$


Note that the electric energy still contains contribution from $\mathbf{B}_{0}$, but that is reduced by the factor $\left(1 / c^{2}\right)$. With these definitions the conservation form of the semi-relativistic MHD equations (eq. 11) can be rewritten as

$$
\begin{gathered}
\frac{\partial \rho}{\partial t}+\nabla \cdot(\rho \mathbf{u})=0 \\
\frac{\partial}{\partial t}\left(\rho \mathbf{u}+\frac{1}{c^{2}} \mathbf{S}_{\mathrm{A}}\right)+\nabla \cdot\left[\rho \mathbf{u u}+\mathbf{I} p+\mathbf{P}_{\mathrm{A}, 1}\right]=\mathbf{j}_{0} \times \mathbf{B}_{0} \\
\frac{\partial \mathbf{B}_{1}}{\partial t}+\nabla \cdot[\mathbf{u B}-\mathbf{B u}]=-\frac{\partial \mathbf{B}_{0}}{\partial t} \\
\frac{\partial e_{1}}{\partial t}+\nabla \cdot\left[\mathbf{u}\left(\frac{1}{2} \rho \mathbf{u}^{2}+\frac{\gamma p}{\gamma-1}\right)+\frac{1}{\mu_{0}} \mathbf{E} \times \mathbf{B}_{1}\right]=-\mathbf{B}_{1} \cdot \frac{\partial \mathbf{B}_{0}}{\partial t}+\mathbf{E} \cdot \mathbf{j}_{0}
\end{gathered}
$$

where

$$
P_{\mathrm{A}, 1}=\frac{1}{\mu_{0}}\left[\mathbf{I}\left(\frac{1}{2} \mathbf{B}_{1}^{2}+\mathbf{B}_{1} \cdot \mathbf{B}_{0}+\frac{1}{2 c^{2}} \mathbf{E}^{2}\right)-\mathbf{B}_{1} \mathbf{B}_{1}-\mathbf{B}_{1} \mathbf{B}_{0}-\mathbf{B}_{0} \mathbf{B}_{1}\right]
$$

The splitting did not modify the continuity equation. In the momentum equation the dominant $B_{0}^{2} \mathbf{I}$ and $\mathbf{B}_{0} \mathbf{B}_{0}$ terms are moved into the source term $\mathbf{j}_{0} \times \mathbf{B}_{0}$, which can be calculated analytically, and it is identically zero if $\mathbf{B}_{0}$ is a force free field. The induction equation is modified in a trivial way, by moving the time derivative of $\mathbf{B}_{0}$ to the right hand side. Again, this term can be calculated analytically, and in the case of a stationary $\mathbf{B}_{0}$ field, it vanishes. The split energy equation is obtained after quite some algebra. Most of the dominant $B_{0}^{2}$ and $\mathbf{E} \times \mathbf{B}_{0}$ terms are eliminated, but the remaining source terms $\mathbf{B}_{1} \cdot \partial \mathbf{B}_{0} / \partial t$ and $\mathbf{E} \cdot \mathbf{j}_{0}$ contain the numerically calculated $\mathbf{B}_{1}$ and $\mathbf{u}$ quantities. In case of a potential $\left(\mathbf{j}_{0}=0\right)$ and/or stationary $\mathbf{B}_{0}$ field one or both energy source terms can be eliminated.

One may add the source terms involving $\nabla \cdot \mathbf{B}$ to the split momentum, induction and/or energy equations (18b) - 18d if the numerical scheme does not keep $\nabla \cdot \mathbf{B}$ exactly zero. Of course, $\nabla \cdot \mathbf{B}=\nabla \cdot \mathbf{B}_{1}$, since the analytic $\mathbf{B}_{0}$ field must be divergence free by definition.

\section{Solution Techniques}

\subsection{Finite-Volume Scheme}

The MHD equations are well suited for finite volume methods when the governing equations are integrated over a computational cell $i$, yielding

$$
\frac{d \mathbf{U}_{i}}{d t}=-\frac{1}{V_{i}} \sum_{\text {faces }} \mathbf{F} \cdot \hat{\mathbf{n}} A-\frac{\mathbf{Q}_{i}}{V_{i}} \sum_{\text {faces }} \mathbf{B} \cdot \hat{\mathbf{n}} A,
$$

where $V_{i}$ is the volume of cell $i, A$ is the surface area of the faces forming the computational cell, $\hat{\mathbf{n}}$ is the unit vector normal to the cell faces, $\mathbf{U}_{i}$ is the cellaveraged conserved solution vector, and $\mathbf{Q}_{i}$ is given by

$$
\mathbf{Q}_{i}=-\left[\begin{array}{c}
0 \\
\frac{1}{\mu_{0}} \mathbf{B}_{i} \\
\mathbf{u}_{i} \\
\frac{1}{\mu_{0}} \mathbf{u}_{i} \cdot \mathbf{B}_{i}
\end{array}\right]
$$


The numerical face fluxes, $\mathbf{F} \cdot \hat{\mathbf{n}}$, are defined in terms of the left and right interface solution states, $\mathbf{U}_{\mathrm{L}}$ and $\mathbf{U}_{\mathrm{R}}$, as follows

$$
\mathbf{F} \cdot \hat{\mathbf{n}}=\mathcal{F}\left(\mathbf{U}_{\mathrm{L}}, \mathbf{U}_{\mathrm{R}}, \hat{\mathbf{n}}\right),
$$

where $\mathbf{U}_{L}$ and $\mathbf{U}_{R}$ are the state vectors at the left and right sides of the interface.

\subsection{TVD-MUSCL}

Because the MHD equations are a system of hyperbolic conservation laws, many of the techniques that have been developed for the Euler equations can be applied relatively straightforwardly. In particular, the high-resolution finite-volume approach of van Leer 69] (i.e. approximate Riemann solver + limited interpolation scheme + multi-stage time-stepping scheme) is perfectly valid. The Rusanov/Lax-Friedrichs approximate Riemann solver can be applied directly; no knowledge of the eigensystem of the MHD equations is required other than the fastest wave speed in the system. A Roe-type scheme [58] can be constructed for non-relativistic MHD, but requires more work, because of the complexity of the eigensystem. In addition, an HLLE-type Riemann solver has been derived by Linde [39]; it is less dissipative than the Rusanov/Lax-Friedrichs scheme, but less computationally intensive than the Roe scheme. Whichever approximate Riemann solver is chosen to serve as the flux function, standard interpolation schemes and limiters can be used to construct a finite-volume scheme.

\subsection{Pressure Positivity}

One added difficulty in solving the MHD equations is that the MHD energy has three components: internal, magnetic and kinetic. Thus, as in gasdynamics, flows with substantially more kinetic energy than internal energy can lead to positivity problems when computing the pressure. Also, in contrast to gasdynamics, regions in which the magnetic field is large can yield similar problems. Conservative and positive HLLE-type schemes for MHD have been described by Janhunen 32 . Another alternative, due to Balsara and Spicer [2], is to use a hybrid scheme: both the conservative energy equation and the entropy equations are solved. Close to shock waves the energy equation is used to obtain the correct weak solution, at other places the more robust and positive entropy equation can be used. A variant of this technique has been implemented in our code.

\section{Controlling $\nabla \cdot \mathrm{B}$}

Another way in which the numerical solution of the MHD equations differs from that of the gasdynamic equations is the constraint that $\nabla \cdot \mathbf{B}=0$. Enforcing this constraint numerically, particularly in shock-capturing codes, can be done in a number of ways, but each way has its particular strengths and weaknesses. Only a brief overview is given below; each of the schemes discussed below is explained more fully in the references cited, and Tóth has published a numerical comparison of many of the approaches for a suite of test cases [66]. 


\subsection{Projection Scheme}

Brackbill and Barnes [12] proposed using a Hodge-type projection to the magnetic field. This approach leads to a Poisson equation that must be solved each time the projection takes place:

$$
\begin{gathered}
\nabla^{2} \phi=\nabla \cdot \mathbf{B} \\
\mathbf{B}_{\text {projected }}=\mathbf{B}-\nabla \phi
\end{gathered}
$$

The resulting projected magnetic field is divergence-free on a particular numerical stencil, to the level of error of the solution of the Poisson equation. While it is not immediately obvious that the use of the projection scheme in conjunction with the fully conservative form of the MHD equations gives the correct weak solutions, Tóth has proven this to be the case [66. The projection scheme has several advantages, including the ability to use standard software libraries for the Poisson solution, its relatively straightforward extension to general unstructured grids, and its robustness. It does, however, require solution of an elliptic equation at each projection step; this can be expensive, particularly on distributed-memory machines.

\subsection{8-Wave Scheme}

Powell [45]46] first proposed an approach based on the symmetrizable form of the MHD equations (eq. 8). In this approach, the source term on the righthand side of eq. (8) is computed at each time step, and included in the update scheme. Discretizing this form of the equations leads to enhanced stability and accuracy, however, there is no stencil on which the divergence is identically zero. In most regions of the flow, the divergence source term is small. However, near discontinuities, it is not guaranteed to be small. In essence, the inclusion of the source term changes what would be a zero eigenvalue of the system to one whose value is $u_{n}$, the component of velocity normal to the interface through which the flux is computed. The scheme is typically referred to as the eight-wave scheme; the eighth wave corresponds to propagation of jumps in the normal component of the magnetic field.

The eight-wave scheme can be thought of as a hyperbolic or advective approach to controlling $\nabla \cdot \mathbf{B}$; symmetrizable form of the equations, eq. (8), are consistent with the passive advection of $\nabla \cdot \mathbf{B} / \rho$. The eight-wave scheme is computationally inexpensive, easy to add to an existing code, and quite robust. However, if there are regions in the flow in which the $\nabla \cdot \mathbf{B}$ source term (eq. 9) is large, the conservation errors can create problems.

\subsection{Constrained Transport}

Several approaches have been developed that have combined a Riemann-solverbased scheme with constrained-transport approach. The constrained-transport 
approach of Evans and Hawley [19] treated the MHD equations in the gasdynamics/electromagnetic-split form of eq. (3a) through 3d . The grid used was a staggered one, and the $\nabla \cdot \mathbf{B}=0$ constraint was met identically, on a particular numerical stencil.

Dai and Woodward [13] and Balsara and Spicer 1] modified the constrainedtransport approach by coupling a Riemann-solver-based scheme for the conservative form of the MHD equations (eq. 5), with a constrained-transport approach for the representation of the magnetic field. In their formulations, this required two representations of the magnetic field: a cell-centered one for the Godunov scheme, and a face-centered one to enforce the $\nabla \cdot \mathbf{B}=0$ condition. Tóth 66 . subsequently showed that these formulations could be recast in terms of a single cell-centered representation for the magnetic field, through a modification to the flux function used.

Advantages of the conservative constrained-transport schemes include the fact that they are strictly conservative and that they meet the $\nabla \cdot \mathbf{B}=0$ constraint to machine accuracy, on a particular stencil. The primary disadvantage of the constrained transport technique is the difficulty in extending them to general grids. Tóth and Roe [67] and Balsara [3] made some progress on this front; they developed divergence-preserving prolongation and restriction operators, allowing the use of conservative constrained-transport schemes on h-refined meshes. De Sterck generalized the constrained transport method to unstructured grids [15].

However, it can also be shown that the conservative constrained-transport techniques lose their $\nabla \cdot \mathbf{B}$-preserving properties if different cells are advanced at different physical time rates 68. This rules out the use of local time-stepping (see Sect. 9.1). Thus, while for unsteady calculations the cost of the conservative constrained-transport approach is comparable to the eight-wave scheme, for steady-state calculations (where one would typically use local time-stepping), the cost can be prohibitive.

\subsection{Diffusive Control}

Some of the most recent work on the $\nabla \cdot \mathbf{B}=0$ constraint has been related to modifying the eight-wave approach by adding a source term proportional to the gradient of $\nabla \cdot \mathbf{B}$ so that the the divergence satisfies an advection-diffusion equation, rather than a pure advection equation. Similar schemes have been used for the Maxwell equations [41. This technique, referred to as diffusive control of $\nabla \cdot \mathbf{B}$, has the same advantages and disadvantages as the eight-wave approach. It is not strictly conservative, but appears to keep the level of $\nabla \cdot \mathbf{B}$ lower than the eight-wave approach does. In other recent work by Dedner et al. 14, a generalized Lagrange-multiplier method has been proposed, incorporating the projection approach, the eight-wave approach, and the diffusive-control approach into a single framework. In addition, they also introduced a hyperbolic correction of $\nabla \cdot \mathbf{B}$ errors. 


\section{Block-Based AMR on Cartesian Grids}

Adaptive mesh refinement techniques that automatically adapt the computational grid to the solution of the governing PDEs can be very effective in treating problems with disparate length scales. Methods of this type avoid underresolving the solution in regions deemed of interest (e.g., high-gradient regions) and, conversely, avoid overresolving the solution in other less interesting regions (lowgradient regions), thereby saving orders of magnitude in computing resources for many problems. For typical solar wind flows, length scales can range from tens of kilometers in the near Earth region to the Earth-Sun distance $\left(1.5 \times 10^{11}\right.$ $\mathrm{m})$, and timescales can range from a few seconds near the Sun to the expansion time of the solar wind from the Sun to the Earth $\left(\sim 10^{5} \mathrm{~s}\right)$. The use of AMR is extremely beneficial and almost a virtual necessity for solving problems with such disparate spatial and temporal scales.

\subsection{Adaptive Blocks}

Borrowing from previous work by Berger and coworkers [568910] and Quirk [47 48], and keeping in mind the desire for high performance on massively parallel computer architectures, a relatively simple yet effective block-based AMR technique has been developed and is used in conjunction with the finite-volume scheme described above. The method has some similarities with the block-based approaches described by Quirk and Hanebutte [48] and Berger and Saltzman [10]. Here the governing equations are integrated to obtain volume-averaged solution quantities within rectangular Cartesian computational cells. The computational cells are embedded in regular structured blocks of equal sized cells. The blocks are geometrically self-similar with dimensions $\tilde{\ell}_{x} \times \tilde{\ell}_{y} \times \tilde{\ell}_{z}$ and consist of $N_{x} \times N_{y} \times N_{z}$ cells, where $\tilde{\ell}_{x}, \tilde{\ell}_{y}$, and $\tilde{\ell}_{z}$ are the nondimensional lengths of the sides of the rectangular blocks and $N_{x}, N_{y}$, and $N_{z}$ are even, but not necessarily all equal, integers. Typically, blocks consisting of anywhere between $4 \times 4 \times 4=64$ and $12 \times 12 \times 12=1728$ cells are used (see Fig. 1). Solution data associated with each block are stored in standard indexed array data structures. It is therefore straightforward to obtain solution information from neighboring cells within a block.

Computational grids are composed of many self-similar blocks. Although each block within a grid has the same data storage requirements, blocks may be of different sizes in terms of the volume of physical space that they occupy. Starting with an initial mesh consisting of blocks of equal size (i.e., equal resolution), adaptation is accomplished by the dividing and coarsening of appropriate solution blocks. In regions requiring increased cell resolution, a "parent" block is refined by dividing itself into eight "children" or "offspring." Each of the eight octants of a parent block becomes a new block having the same number of cells as the parent and thereby doubling the cell resolution in the region of interest. Conversely, in regions that are deemed overresolved, the refinement process is reversed, and eight children are coarsened and coalesced into a single parent block. 

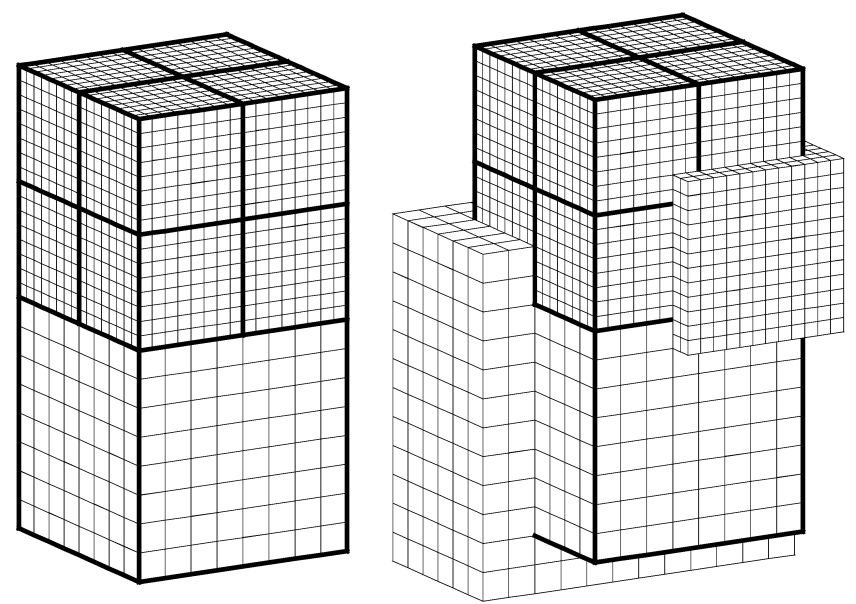

Fig. 1. (left) Self-similar blocks used in parallel block-based AMR scheme. (right) Selfsimilar blocks illustrating the double layer of ghost cells for both coarse and fine blocks.

In this way, the cell resolution is reduced by a factor of 2. Standard multigridtype restriction and prolongation operators are used to evaluate the solution on all blocks created by the coarsening and division processes, respectively.

Two neighboring blocks, one of which has been refined and one of which has not, are shown in Fig. 1. Any of the blocks shown in Fig. 1 can in turn be refined, and so on, leading to successively finer blocks. In the present method, mesh refinement is constrained such that the cell resolution changes by only a factor of 2 between adjacent blocks and such that the minimum resolution is not less than that of the initial mesh.

In order that the update scheme for a given iteration or time step can be applied directly to all blocks in an independent manner, some additional solution information is shared between adjacent blocks having common interfaces. This information is stored in an additional two layers of overlapping "ghost" cells associated with each block as shown in Fig. 1 At interfaces between blocks of equal resolution, these ghost cells are simply assigned the solution values associated with the appropriate interior cells of the adjacent blocks. At resolution changes, restriction and prolongation operators, similar to those used in block coarsening and division, are employed to evaluate the ghost cell solution values. After each stage of the multistage time-stepping algorithm, ghost cell values are reevaluated to reflect the updated solution values of neighboring blocks. With the AMR approach, additional interblock communication is also required at interfaces with resolution changes to strictly enforce the flux conservation properties of the finite-volume scheme [5,68]. In particular, the interface fluxes computed on more refined blocks are used to correct the interface fluxes computed on coarser neighboring blocks so as to ensure that the fluxes are conserved across block interfaces. 


\subsection{Data Structure}

A hierarchical tree-like data structure with multiple "roots," multiple "trees," and additional interconnects between the "leaves" of the trees is used to keep track of mesh refinement and the connectivity between solution blocks. This interconnected "forest" data structure is depicted in Fig. 2. The blocks of the initial mesh are the roots of the forest, which are stored in an indexed array data structure. Associated with each root is a separate "octree" data structure that contains all of the blocks making up the leaves of the tree which were created from the original parent blocks during mesh refinement. Each grid block corresponds to a node of the tree. Traversal of the multitree structure by recursively visiting the parents and children of solution blocks can be used to determine block connectivity. However, in order to reduce overhead associated with accessing solution information from adjacent blocks, the neighbors of each block are computed and stored directly, providing interconnects between blocks in the hierarchical data structure that are neighbors in physical space.

One of the advantages of the preceding hierarchical data structure is that it is relatively easy to carry out local mesh refinement at anytime during a calculation. If, at some point in a computation, a particular region of the flow is

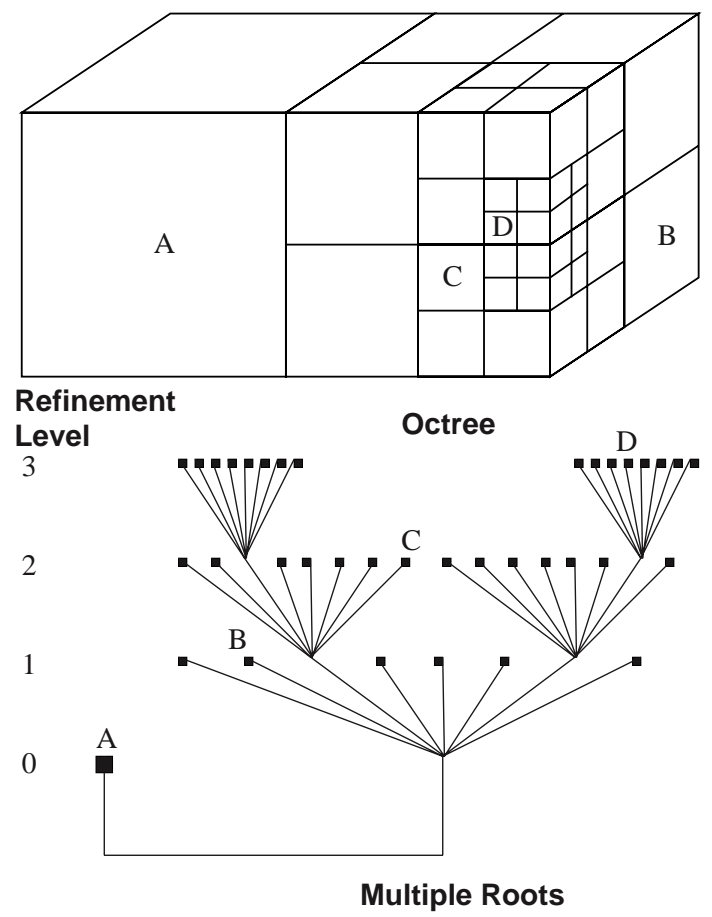

Fig. 2. Solution blocks of a computational mesh with three refinement levels originating from two initial blocks and the associated hierarchical multiroot octree data structure. Interconnects to neighbors are not shown. 
deemed to be sufficiently interesting, better resolution of that region can be attained by refining the solution blocks in that region, without affecting the grid structure in other regions of the flow. Reducing the grid resolution in a region is equally easy. There is no need for completely remeshing the entire grid and recalculating block connectivity every time a mesh refinement is performed. Although other approaches are possible, in BATSRUS the coarsening and division of blocks are directed using multiple physics-based refinement criteria [43|44|46]. In particular, decisions as to when to refine or coarsen blocks are made based on comparisons of the maximum values of various local flow quantities determined in each block to specified refinement threshold values. Note that the refinement thresholds are dynamically adjusted so as to exercise some control over the total numbers of blocks, and hence cells, used in a calculation. We also note that other refinement criteria can also be used, such as a combination of estimated numerical errors.

An example illustrating the adaptation of the block-based Cartesian mesh to an evolving solution is shown in Fig. 3 which shows the grid at four different instances in time for an unsteady calculation and depicts both the solution blocks (thick lines) and computational cells (thin lines) of the evolving grid. As noted
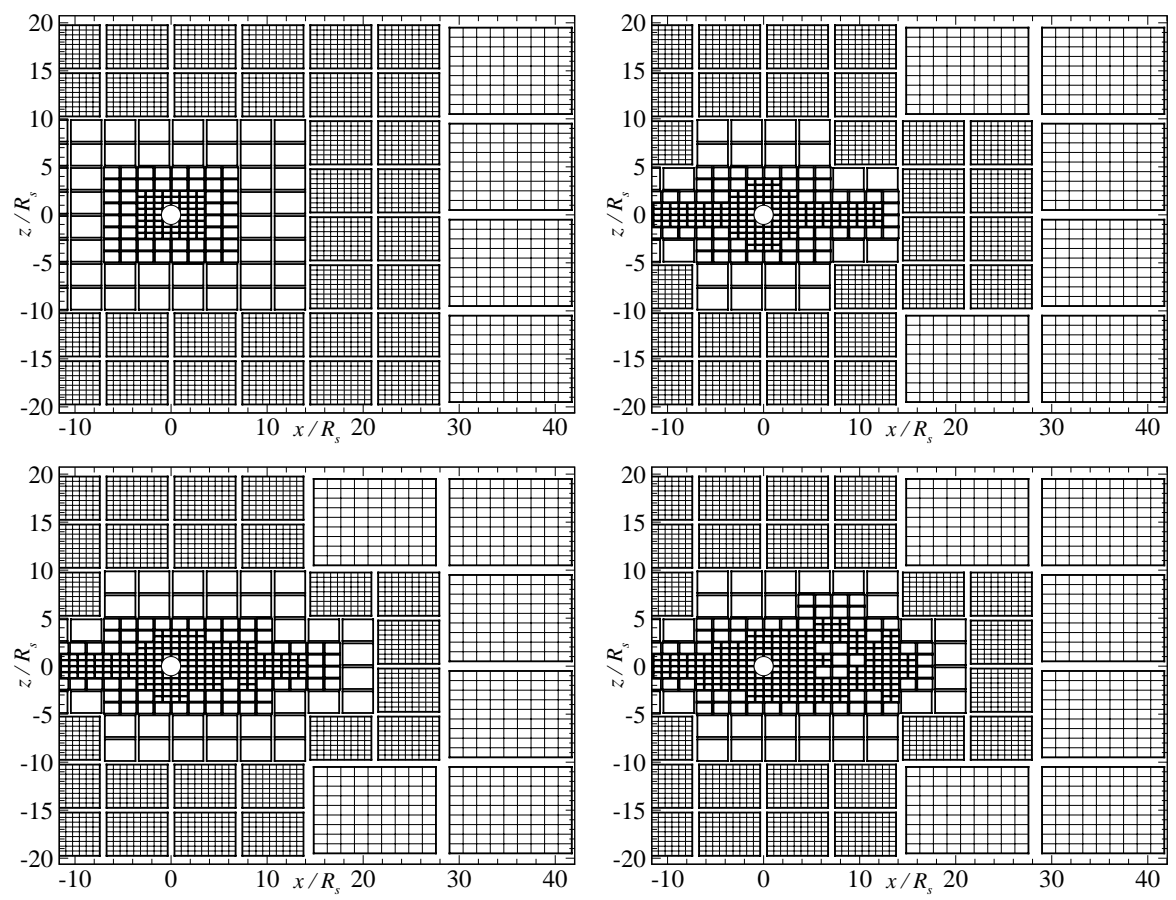

Fig. 3. Evolution of a computational mesh illustrating grid adaptation in response to changes in the numerical solution. Cross sectional cuts through a 3-D grid are shown for a solar wind calculation at four different instances in time. The computational cells are not shown for the smaller blocks. 
above, each level of refinement in the grid introduces cells that are smaller by a factor of 2 in each dimension from those one level higher in the grid. Typically, calculations may have 10-15 levels of refinement; some calculations may have more than 20 levels of refinement. In the case of 20 levels of refinement, the finest cells on the mesh are more than one million times $\left(2^{20}\right)$ smaller in each dimension than the coarsest cells. The block-based AMR approach described above has many similarities to the cell-based method proposed by De Zeeuw and Powell [16]. Although the block-based approach is somewhat less flexible and incurs some inefficiencies in solution resolution as compared to a cell-based approach, the block-based method offers many advantages over a cell-based technique when parallel implementations of the algorithms are considered and performance issues are taken into account. As will be discussed below, the block adaptation readily enables domain decomposition and effective load balancing and leads to low communication overhead between solution cells within the same block.

\section{Parallel Implementation}

The parallel block-based AMR solver was designed from the ground up with a view to achieving very high performance on massively parallel architectures. The underlying upwind finite-volume solution algorithm, with explicit time stepping, has a very compact stencil and is therefore highly local in nature. The hierarchical data structure and self-similar blocks make domain decomposition of the problem almost trivial and readily enable good load-balancing, a crucial element for truly scalable computing. A natural load balancing is accomplished by simply distributing the blocks equally amongst the processors. Additional optimization is achieved by ordering the blocks using the Peano-Hilbert space filling curve to minimize inter-processor communication. The self-similar nature of the solution blocks also means that serial performance enhancements apply to all blocks and that fine grain parallelization of the algorithm is possible. The parallel implementation of the algorithm has been carried out to such an extent, that even the grid adaptation is performed in parallel.

Other features of the parallel implementation include the use of FORTRAN 90 as the programming language and the message passing interface (MPI) library for performing the interprocessor communication. Use of these standards greatly enhances the portability of the code and leads to very good serial and parallel performance. The message passing is performed in an asynchronous fashion with gathered wait states and message consolidation.

Implementation of the algorithm has been carried out on Cray T3E supercomputers, SGI and Sun workstations, on Beowulf type PC clusters, on SGI shared-memory machines, on a Cray T3D, and on several IBM SP2s. BATSRUS nearly perfectly scales to 1,500 processors and a sustained speed of 342 GFlops has been attained on a Cray T3E-1200 using 1,490 PEs. For each target architecture, simple single-processor measurements are used to set the size of the adaptive blocks. The scaling of BATSRUS on various architectures is shown in Fig. 4. 


\section{BATS-R-US Code Speedup on Different Architectures}

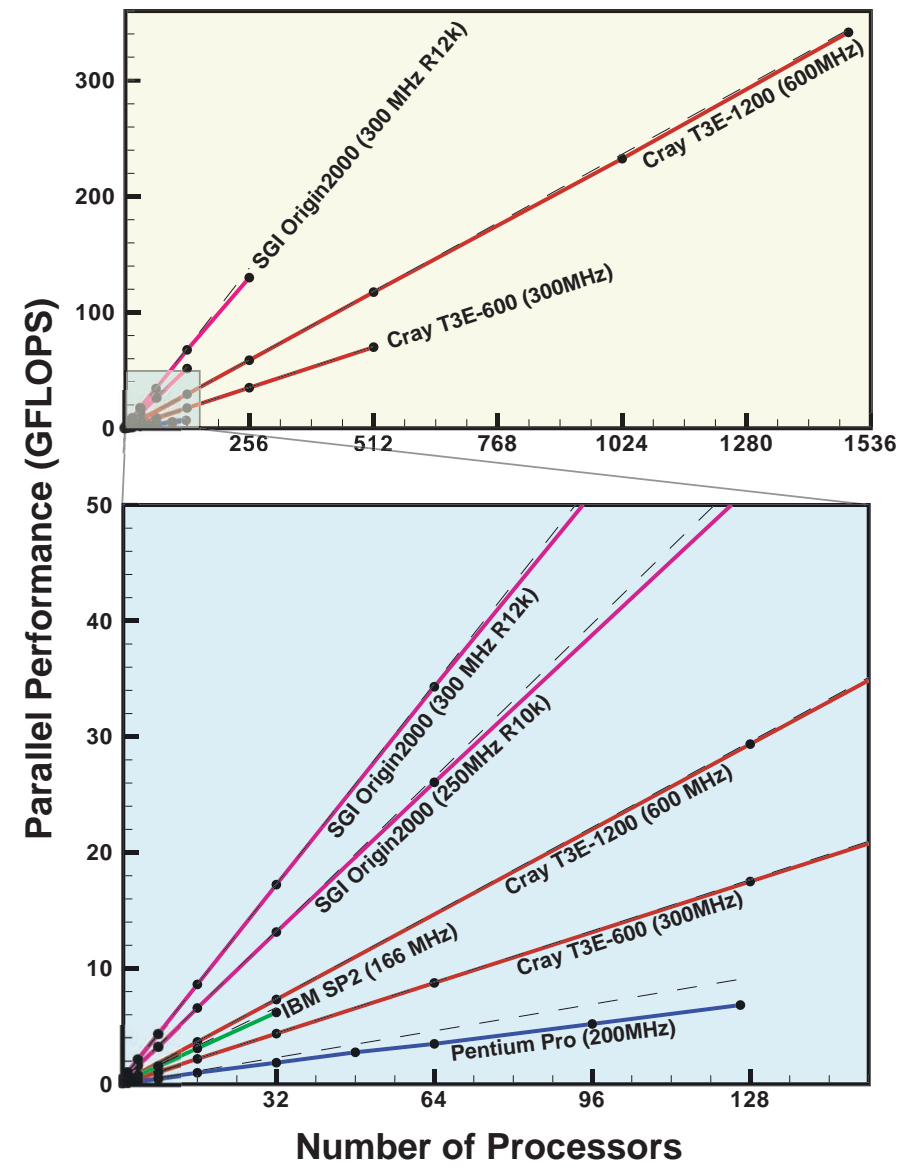

Fig. 4. Parallel speedup of BATSRUS on various architectures. Black dashed lines represent perfect scaling from single node performance.

\section{Time to Solution}

Since a major goal of global space plasma simulations is the creation of a predictive space weather tool, wallclock time to solution is a paramount issue. In particular, a predictive model must run substantially faster than real time. From the starting point - the observation of a solar event, to the ending point - postprocessing the data from a simulation based on the initial conditions derived from the observations, a simulation must be accomplished rapidly to be of use.

The main limitation of the present generation of global space plasma codes is the explicit time stepping algorithm. Explicit time steps are limited by the Courant-Friedrichs-Lewy (CFL) condition, which essentially ensures that no information travels more than a cell size during a time step. This condition rep- 
resents a non-linear penalty for highly resolved calculations, since finer grid resolution not only results in more computational cells, but also in smaller time steps.

In global MHD simulations of space plasmas the CFL condition is controlled by two factors: (1) the smallest cell size in the simulation, and (2) the fast magnetosonic speed in high magnetic field, low plasma density regions. In a typical magnetosphere simulation with a smallest cell size of about $0.25 R_{\mathrm{E}}$ the CFL condition limits the time step to about $10^{-2} \mathrm{~s}$. This small step is primarily controlled by the high fast magnetosonic speed (due to the high Alfvén speed) in the near-Earth region.

\subsection{Local Time Stepping}

In the local time stepping approach the time step in each cell of the computational domain is determined by the local stability condition. The flow variables in cell $i$ are advanced from time step $n$ to time step $n+1$ as

$$
U_{i}^{n+1}=U_{i}^{n}+\Delta t_{i}^{n}(-\nabla \cdot \mathbf{F}+Q)_{i}
$$

where the local time step is determined from the stability condition. Here $U$ represents the conservative state vector, $\mathbf{F}$ is the flux diad and $Q$ is the source term. In case of ideal MHD, the time step is determined by the CFL condition

$$
\Delta t_{i}^{n}=C \frac{\Delta x_{i}}{c_{i}^{\text {fast }}+\left|u_{i}\right|}
$$

where $C<1$ is the Courant number and $c_{i}^{\text {fast }}$ is the fast speed in cell $i$. In more than 1D the sum of the speeds in all directions should be taken in the denominator.

Note that this technique is different from "sub-cycling" when cells are advanced at the same physical time rate, but the number of time-steps taken by individual cells varies. For example, in adaptive grids it is customary to set the time-step to be inversely proportional to the size of the cell, so that a finer cell typically makes two half time-steps while the coarser cell makes only one full time-step. In this method the time-steps are still determined by a global stability condition as opposed to local time-stepping where time-steps are set on a cell-by-cell basis.

It is easy to see from (24) that the steady state solution, if it exists, satisfies

$$
0=(-\nabla \cdot \mathbf{F}+Q)_{i}
$$

since in steady state $U_{i}^{n+1}=U_{i}^{n}$ and we can simplify with the time step $\Delta t_{i}^{n}$ which is always a positive number. Consequently the steady state solution is independent of the time step, and it does not matter if it is local or global.

The above proof assumes that the steady state is fully determined by the boundary conditions. This is a non-trivial assumption, because the MHD equations are non-linear, and there is no mathematical theorem that would guarantee 
the existance and uniquness of the steady state solution. In practice, magnetosphere simulations seem to converge to the same solution independent of the initial conditions or the time integration scheme.

The applicability of the local time-stepping technique in a given scheme primarily depends on the evolution of $\nabla \cdot \mathbf{B}$. In some methods even if $\nabla \cdot \mathbf{B}=0$ initially, the numerical transients towards steady state will destroy this property if the local time stepping is applied. For instance, it can be shown that the constrained transport scheme cannot be combined with local time stepping.

\subsection{Implicit Time-Stepping}

In BATSRUS we have a number of time stepping algorithms implemented. The simplest and least expensive scheme is a multistage explicit time stepping, for which the time step is limited by the CFL stability condition. We have also implemented an unconditionally stable fully implicit time stepping scheme 65 36]. The second order implicit time discretization (BDF2) requires the solution of a non-linear system of equations for all the flow variables. This can be achived by the Newton-Krylov-Schwarz approach: a Newton iteration is applied to the nonlinear equations; a parallel Krylov type iterative scheme is used to solve the linear systems; the convergence of the Krylov solver is accelerated with a Schwarz type preconditioning. In our implementation the Krylov solver is BiCGSTAB, and a modified block incomplete LU (MBILU) preconditioner is applied on a block by block basis. Since every block has a simple Cartesian geometry, the preconditioner can be implemented very efficiently. The resulting implicit scheme requires about 20-30 times more CPU time per time step than the explicit method, but the physical time step can be 1,000 to 10,000 times larger. This implicit algorithm has a very good parallel scaling due to the Krylov scheme and the block by block application of the preconditioner.

In BATSRUS, we can combine explicit and implicit time stepping. Magnetosphere simulations include large volumes where the Alfvén speed is quite low (tens of $\mathrm{km} / \mathrm{s}$ ) and the local CFL number allows large explicit time steps (tens of seconds to several minutes). In these regions implicit time stepping is a waste of computational resources. Since the parallel implicit technique we use is fundamentally block based we only treat those blocks implicitly where the CFL condition would limit the explicit time step to less than the selected time step (typically $\sim 10 \mathrm{~s}$ ). Needless to say, this combined explicit-implicit time stepping represents more computational challenges (such as separate load balancing of explicit and implicit blocks). Overall, this solution seems to be a very promising option, but other potential avenues need to explored before one makes a final decision about the most efficient time-stepping algorithm for space MHD simulations. These questions will be discussed in an upcoming paper 68.

\section{Ionosphere-Magnetosphere Coupling}

The state of the magnetosphere is controlled by conditions in the solar wind and in the ionosphere. Solar wind conditions are imposed as boundary conditions at 
the outer boundaries of the simulation domain. Even though the numerical procedures used to impose these boundary conditions can be quite sophisticated, the distant solar wind (in all directions) is assumed to be unaffected by the presence of the magnetosphere. This assumption makes the outer boundary conditions relatively simple (at least in principle).

The ionosphere-magnetosphere (I-M) coupling, on the other hand, is a highly non-linear two-way interaction which strongly affects the large-scale behavior of both domains. Self-consistent global magnetosphere models include some kind of dynamic ionosphere model which interacts with the magnetosphere and provides ionospheric boundary conditions actively responding to changing magnetospheric conditions.

While mass exchange between the ionosphere and the magnetosphere is undoubtedly of major importance, the dominant component of I-M coupling is a system of field-aligned currents (FACs) connecting the magnetosphere and the high-latitude ionosphere. These FACs carry momentum (electromagnetic stress) and energy (Poynting flux) along stretched magnetic field lines connecting the ionosphere and the magnetosphere. Self-consistent global magnetosphere models need to describe the generation and closure of these FACs through appropriate boundary conditions and embedded non-MHD models.

The most important current systems coupling the ionosphere and the magnetosphere are the so called Region 1 and Region 2 currents. Region 1 FACs, flowing near the open-closed magnetic field boundary, connect the magnetopause current to the ionosphere where they close through ionospheric Pedersen currents. Region 2 FACs flow along closed magnetic field lines and they connect to the ionosphere at lower magnetic latitudes than the Region 1 current. Region 2 currents are generated in the inner part of the plasma sheet and in the ring current region.

The generation of Region 2 currents can not be adequately described by an MHD model alone. The main physical process leading to the generation of these currents is the gradient and curvature drifts of hot magnetospheric particles. Since single-fluid MHD represents all particles with a single bulk velocity (obtained as a mass weighted average), it provides an inaccurate representation of the important drift processes (in reality electrons and ions drift in opposite directions, and ions with different energy and equatorial pitch-angle have very different drift velocities). As a result of this limitation of the single-fluid treatment, one needs to embed an accurate drift model into the global MHD code in order to get physically consistent Region 2 currents.

BATSRUS has been coupled to two ionosphere models to provide ionospheric current closure (the user can choose from the two available models) and a drift model to account for the generation and closure of FACs.

\subsection{Electrostatic Ionosphere}

The simplest I-M coupling procedure involves a height integrated electrostatic ionosphere model. In this case the MHD code has an inner boundary at a radius of $R_{\mathrm{B}}$ (for explicit time-stepping we typically use $R_{\mathrm{B}}=2.5 R_{\mathrm{E}}$ ). At this inner 
boundary, the plasma density, temperature, and velocity are specified. In addition, the magnetic field is allowed to float (zero gradient of the normal component), so currents can flow along the boundary. The velocities which are imposed on the boundary are calculated in the ionosphere in a three step process:

1. field aligned currents are calculated from the curl of the magnetic field at $R_{\mathrm{B}}+1 R_{\mathrm{E}}$, and these are mapped down to the ionosphere,

2. a height-integrated ionospheric conductance pattern is generated and the ionospheric potential is calculated from the equation:

$$
j_{\mathrm{R}}\left(R_{\mathrm{E}}\right)=\left[\nabla_{\perp} \cdot(\Sigma \cdot \nabla \psi)_{\perp}\right]_{R=R_{\mathrm{E}}}
$$

which describes the relationship between the height integrated conductance tensor, $\Sigma$, the ionospheric potential, $\psi$, and the radial component of the current, $j_{\mathrm{R}}$; and finally

3. the electric potential is mapped out along field lines to the inner boundary at $R_{\mathrm{B}}$ where electric fields and velocities are generated. The corotation velocity field is added to the ionosphere generated velocity field.

The details of our conductance model as well as some simulations results using this method are given in 525355 .

\subsection{BATSRUS-TIEGCM Coupling 54}

As our physical understanding of the magnetosphere - ionosphere system increases, our need to have more accurate models of the coupled system also grows. Recently we developed a coupled model of the magnetosphere - ionosphere thermosphere system. The model is based on BATSRUS [46] and the thermosphere - ionosphere - electrodynamics general circulation model (TIEGCM) 50] which has been used in numerous studies of the ionosphere - thermosphere system.

The TIEGCM solves for the thermospheric and ionospheric composition, temperature, and winds. It solves for mass mixing ratios of the neutral major species $\mathrm{O}_{2}, \mathrm{~N}_{2}$, and $\mathrm{O}$ using full transport and chemistry, while the minor species $\mathrm{N}\left({ }^{2} D\right), \mathrm{N}\left({ }^{4} S\right), \mathrm{NO}, \mathrm{He}$, and Ar are obtained by assuming that they are in local equalibrium with the major species. For the ions, the $\mathrm{O}_{2}^{+}$dynamics are considered, while the species $\mathrm{N}_{2}^{+}, \mathrm{NO}^{+}$, and $\mathrm{N}$ are considered to be in local equalibrium with $\mathrm{O}_{2}^{+}$. The TIEGCM is a full 3-dimensional code with $5^{\circ}$ latitude by $5^{\circ}$ longitude by 0.5 scale height altitude cells. There are 29 pressure levels in the model such that the simulation spans from $\sim 95 \mathrm{~km}$ to $650 \mathrm{~km}$ in altitude.

The electrodynamics within the TIEGCM focuses on the middle and lower latitudes, with a self consistent calculation of the interaction between the neutral winds, currents, and electric fields. At the high latitudes, an external electric field is assumed, and the neutral winds do not affect this field (i.e. there is no ion drag). At all latitudes, the neutral winds are coupled with ion flow through ion drag terms. A transition region exists where the model linearly scales the electric field between the high and middle latitude regions. The electrodynamics of the 
TIEGCM are solved on a magnetic apex grid based on the 1985 International Geomagnetic Reference Field (IGRF) 51.

The main auroral electron precipitation is specified at high latitudes using an external model [57. In addition to the main oval, a polar cap precipitation is specified as well as a spatially limited cusp precipitation. These precipitation patterns are used to generate 3 -d ionization rates, and therefore strongly control the electron density at high latitudes.

The ionosphere is coupled to the MHD code in a similar manner as in the case of an electrostatic ionosphere (see section 10.1). The field aligned currents at $3.5 R_{\mathrm{E}}$ are derived from $\nabla \times \mathbf{B}$. These currents are mapped down to the ionosphere using the intrinsic magnetic field within the code, and the currents are scaled by the ratio of the magnetic field in the ionosphere to that in the magnetosphere. The currents are combined with a conductance pattern to produce a potential pattern, which is mapped up to the magnetospheric inner boundary at 2.5 $R_{\mathrm{E}}$. Corotation velocities are determined and added to the convection velocities derived from the potential pattern. These velocities are applied as the inner boundary condition on the MHD solution.

BATSRUS provides the ionospheric potential pattern and electron precipitation pattern for TIEGCM. The electon precipitation is used to determine the nightside ionization in calculating the electron density. The electron density, combined with the densities of different neutral consituents, is subsequently used to determine the densities of different ion species. Collision rates between ions and neutrals are then calculated. These collision rates specify the acceleration of the neutral wind and the different conductivities.

In the low- and mid-latitude region, the neutral winds drive the dynamo calculation. This is done by taking a field-line integral of the neutral wind velocity multiplied by the conductivity. The divergence of this quantity is considered to be a quasi-neutral wind field aligned current, and is used for the calculation of the low- and mid-latitude dynamo in the TIEGCM. This quantity is also fed back into the MHD potential solver, where it is subtracted from the magnetospheric FACs. The neutral wind driven field aligned currents above $60^{\circ}$ are considered in the calculation of the MHD potential, while those below are ignored.

The conductances are not completely consistent between the two models. The reasoning for not using the TIEGCM calculated conductances in the MHD model is that the grid size in the TIEGCM ( $5^{\circ}$ latitude by $5^{\circ}$ longitude) is too large. The auroral zone is ill represented by the TIEGCM. Because the particle precipitation is calculated by the MHD model, and the conductances derived from the Robinson formula [56] closely match those derived by models such as the TIEGCM, it was decided that it is better to use the high resolution conductances from the MHD model than the low resolution conductances from the TIEGCM. Once the resolution of the TIEGCM is increased, a more self-consitent coupling can occur.

The BATSRUS-TIEGCM coupling has been fully implemented and tested the first results obtained with the coupled model have also been reported [54]. 


\subsection{BATSRUS-RCM Coupling [17]}

The Rice Convection Model (RCM) calculates the dynamic behavior of the particles and the electric fields and currents in the Earth's inner magnetosphere (the region of closed magnetic lines). The physics of this region is complicated, because it contains overlapping particle distributions with a wide range of energies and characteristics. These different coexisting particle populations cannot be treated as a single fluid, because they all move differently. The RCM represents the particles in terms of 30-200 separate fluids. Its equations and numerical methods have been specifically designed for accurate treatment of the inner magnetosphere 31,29,71/18, including the flow of electric currents along magnetic field lines to and from the conducting ionosphere. The RCM does its primary calculations on a 2D grid on a spherical shell in the ionosphere. Values in the magnetosphere are computed by mapping out along magnetic field lines. The ionospheric grid is fine, typically about 0.5 degrees latitude in the auroral zone. The RCM computes these currents and the associated electric fields self-consistently.

The essential limitation of the RCM is that it only describes the inner magnetosphere: one needs to specify its connections to the outer magnetosphere and to the ionosphere and thermosphere through externally specified magnetic field models and particle source/sink boundary conditions. This can be done by coupling RCM to a global MHD code which dynamically provides these inputs and uses the results of the drift physics represented by RCM to improve the MHD solution.

RCM calculates the Region 2 field-aligned currents using the Vasyliunas formula 70. This formula (which is also valid in the MHD limit) relates the field aligned current in slow flow regions to the cross product of the pressure gradient and the gradient of the magnetic flux tube volume. The pressure is calculated as a sum of partial pressures of the large number of "species," while the flux tube volume is calculated by integrating the flux tube cross secton $(\propto 1 / B)$ along magnetic field lines. In dynamic calculations (when the magnetic field is changing with time) the flux tube volume calculation is the most time consuming component of the entire simulation.

As a first step in coupling BATSRUS and RCM the Rice Convection Model was reformulated and a modern advection module was incorporated. Specifically the following changes were made:

1. Make the RCM code and its data structure compatible with BATSRUS.

2. Introduce MPI parallelization into RCM.

3. Introduce a new numerical scheme for solving the advection equations to achieve second-order accuracy and to handle time-dependent moving boundary conditions.

4. Improve other numerical algorithms (e.g., Birkeland current calculation.)

These changes in RCM already resulted in new physics, since the updated RCM code has more powerful capabilities than the original 60]. 
In the second step we introduced full, two-way coupling between BATSRUS and RCM. BATSRUS provides to RCM the full near-earth magnetic field (all closed field lines) as well as the flux-tube volume for the magnetic field line originating at each RCM grid point in the ionosphere. In addition, BATSRUS also provides the high-latitude ionospheric electric potential distribution and the particle sources at the outer boundaries at RCM. In return, RCM provides the hot plasma pressure averywhere in the closed field line region, which is used to to "nudge" the MHD pressure towards the RCM pressure (this is done through a source term).

The most computationally challenging aspect of this two-way coupling procedure is mapping between the 2D RCM and 3D MHD grids and the related calculation of the flux-tube volume for the RCM grid. This calculation is extremely time consuming if one uses standard field-line tracing routines and methods (in case of a reasonably refined grid this step might take hours of CPU time). We developed a block-based parallel ray-tracing algorithm which is about $10^{4}$ times faster than the "brute force" method using a modest number of processors (about 32) [17]. This new algorithm makes the frequent two-way information exchange possible between BATSRUS and RCM.

The self-consistently coupled BATSRUS-RCM code has been recently tested and the results are being published [17]. The coupled code is capable describing the I-M coupling in a self-consistent manner, including the Region 1 and Region 2 FAC systems.

\section{Applications}

BATSRUS has been extensively applied to global numerical simulations of the inner heliosphere including CME propagation [24|26], the coupled terrestrial magnetosphere-ionosphere 236162 , and the interaction of the heliosphere with the interstellar medium [38. In addition, it has also been successfully applied to a host of planetary problems ranging from comets [22 27], to Mercury 34], Venus [4, Mars [40, Saturn 28, to planetary satellites 3335.

In this section we briefly summarize our most ambitious space weather simulation so far, in which we used BATSRUS to simulate an entire space weather event, from its generation at the Sun through the formation and evolution of a $\mathrm{CME}$, to its interaction with the magnetosphere-ionosphere system [2426]. In this simulation we resolved multiple spatial and temporal scales and took advantage of frequent grid refinements and coarsening to follow the CME through interplanetary space. The total number of cells varied between 800,000 and 2 million as the solution evolved. The simulation used 13 levels of grid refinement. The simulation ran faster than real time on a 512 node Cray T3E-600 supercomputer. This simulation demonstrates that we have the necessary experience to undertake the research outlined in this proposal.

Here we only show a few highlights of this simulation. The detailed results have been published in JGR-Space Physics [26]. 


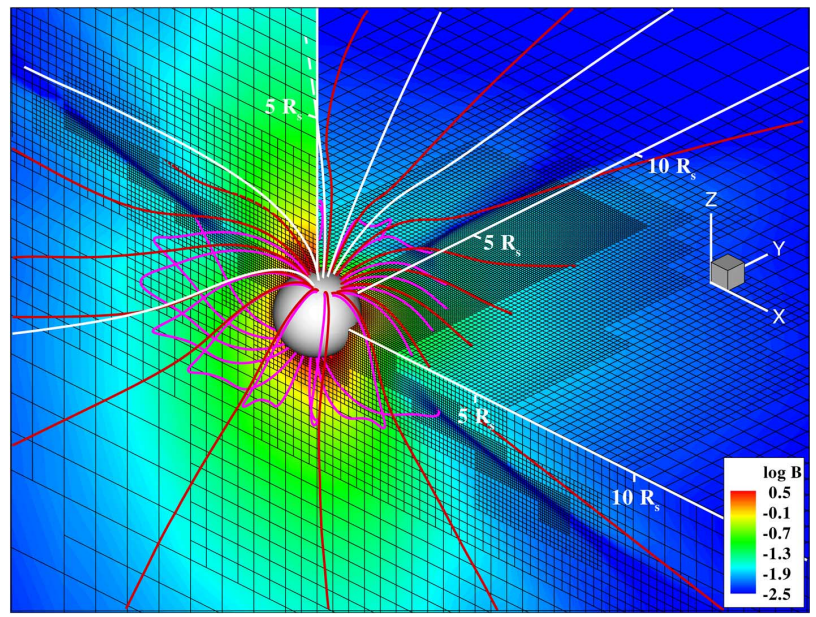

Fig. 5. 3D representation of the steady-state solar wind solution. The shading represents $\log |\mathbf{B}|$ in the $(x, z)$ - and $(x, y)$-planes. The thin black lines are the computational mesh and the thick solid lines are magnetic field lines: grey denotes the last closed field lines, black is open field lines expanding to the interplanetary medium just above the heliospheric current sheet, and finally, white lines show open magnetic field lines in the $(y, z)$-plane.

A steady state solar wind was obtained in the corotating frame for a tilted rotating Sun. The intrinsic magnetic field was approximated by the superposition of a tilted (with respect to the rotation axis) octupole and dipole. Figure 5 depicts a three-dimensional representation of the predicted pre-event steady-state solar wind solution in the vicinity of the Sun. The narrow dark region shown in Fig. 5 , which also coincides with regions of higher mesh refinement, corresponds to the beginning of the heliospheric current sheet. Due to the combined effects of magnetic tilt and solar rotation, the current sheet is tilted with respect to the rotation axis, and deformed, and resembles a "ballerina skirt."

Figure 6] shows a 3D representation of the magnetic field configuration 9 hours after the initiation of the CME. The density enhancement first leads to the "filling" of the closed magnetic field lines with additional plasma and subsequent expansion of the closed field line region. One can see that the closed field lines become greatly stretched by the outward moving plasma. This is due to the fact that the plasma $\beta$ (the ratio of the kinetic and magnetic pressures) is quite large and the magnetic field is "carried" by the outward moving plasma. We also note the decrease of magnetic field strength behind the leading edge of the outward moving disturbance.

The dynamic response of the global magnetosphere to the changing solar wind conditions produced by the density-driven CME was also computed as part of this simulation. The global magnetospheric configuration for quiet-time southward IMF conditions is shown in Fig. 7, During the event the solar wind velocity remained nearly radial with the speed gradually decreasing from about 


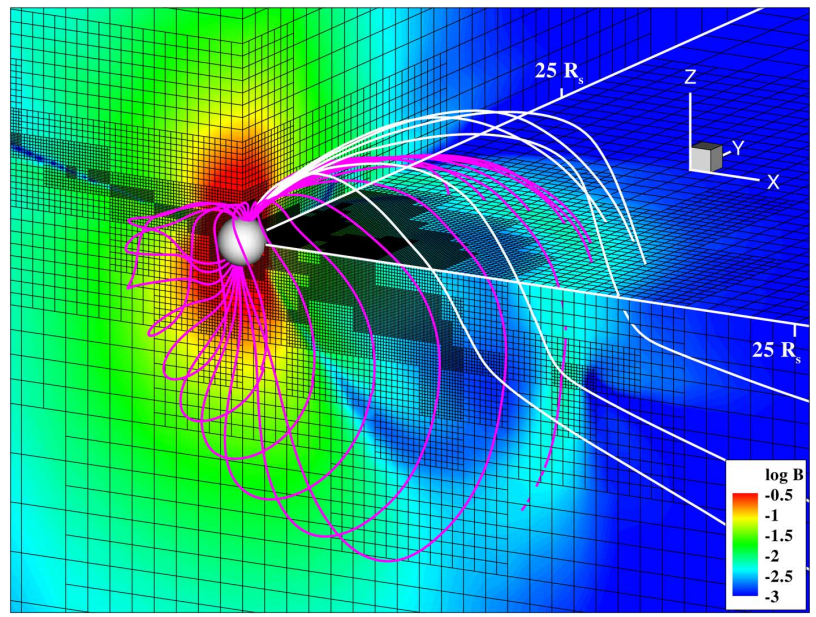

Fig. 6. 3D representation of magnetic field lines 9 hours after the initiation of a CME. Grayscale represents $\log (B)$, white lines are open magnetic field lines, grey lines represent magnetic field lines with both ends connected to the Sun.

$550 \mathrm{~km} / \mathrm{s}$ to about $450 \mathrm{~km} / \mathrm{s}$. The solar wind dynamic pressure increased from its pre-CME value of $2.25 \mathrm{nP}$ (at $t=72 \mathrm{hrs)}$ to $4.6 \mathrm{nP}$ at the peak of the event.

The ionospheric potential and convection patterns also change during the CME event. The ionospheric convection shows the two-cell pattern of ionospheric convection typical for southward-type IMF conditions. The convection pattern is also "twisted" due to the presence of a non-zero IMF $B_{y}$ component. The most important change in the ionosphere is the doubling of the cross-cap potential drop from $30 \mathrm{kV}$ at $70.5 \mathrm{~h}$ to $60 \mathrm{kV}$ some 27 hours later.

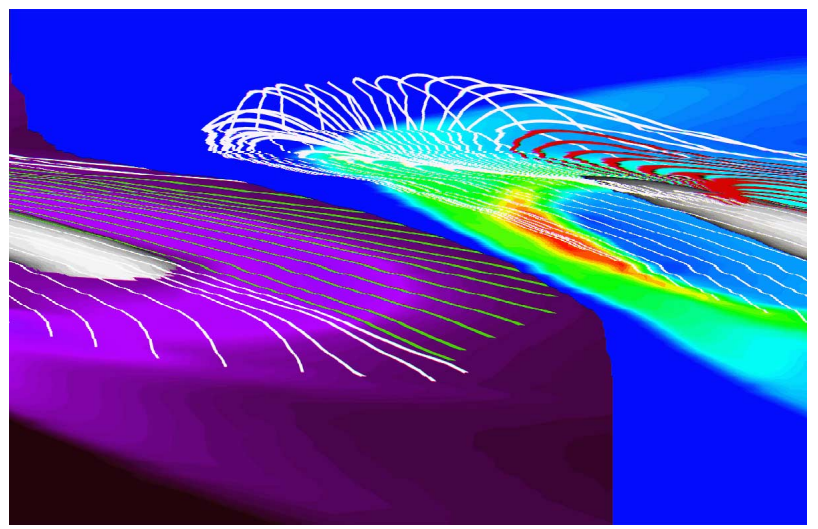

Fig. 7. 3D representation of the last closed terrestrial field lines for southward IMF conditions. White field lines form the dayside magnetopause, while black ones map to the magnetotail. The greyscale represents normalized thermal pressure. 
Overall, this simulated space weather event was not very geoeffective. It is expected that we will be able to generate more geoeffective CMEs with the help of more realistic explosive event generation modules. This simulation, however, demonstrates the present capabilities of BATSRUS.

\section{Acknowledgements}

This work was supported by DoD MURI grant F49620-01-1-0359, NSF KDI grant NSF ATM-9980078, NSF CISE grant ACI-9876943, and NASA AISRP grant NAG5-9406. G. Tóth is partially supported by the Education Ministry of Hungary (grant No. FKFP-0242-2000). We also acknowledge the contributions of the developers of TIEGCM and RCM to the coupled magnetosphere model. In particular, the contributions of Ray Roble, Stanislav Sazykin and Richard Wolf are acknowledged and appreciated.

\section{References}

1. D. S. Balsara and D. S. Spicer: J. Comput. Phys., 149, 270 (1999)

2. D. S. Balsara and D. S. Spicer: J. Comput. Phys., 148, 133 (1999)

3. D. Balsara: J. Comput. Phys., 174, 614 (2001)

4. R. Bauske, A. F. Nagy, T. I. Gombosi, D. L. De Zeeuw, K. G. Powell and J. G. Luhmann: J. Geophys. Res., 103, 23,625 (1998)

5. M. J. Berger: 'Adaptive Mesh Refinement for Hyperbolic Partial Differential Equations'. Interanl report at Stanford Univ., Stanford, Calif., 1982

6. M. J. Berger: J. Comput. Phys., 53, 484 (1984)

7. M. J. Berger and A. Jameson: AIAA Journal, 32, 561 (1985)

8. M. J. Berger and P. Colella: J. Comput. Phys., 82, 67 (1989)

9. M. J. Berger and R. J. LeVeque: 'An adaptive Cartesian mesh algorithm for the Euler equations in arbitrary geometries'. In Proc. 9th AIAA Computational Fluid Dynamics Conference (AIAA Paper No. 89-1930, Buffalo, NY 1989)

10. M. J. Berger and S. Saltzman: "Appl. Numer. Math.", 14, 239 (1994)

11. Boris, J. P.: 'A physically motivated solution of the Alfvén problem'. Internal report at Naval Research Laboratory, NRL Memorandum Report 2167, Washington, D.C., 1970

12. J.U. Brackbill and D.C. Barnes: J. Comput. Phys., 35, 426 (1980)

13. W. Dai and P. R. Woodward: J. Comput. Phys., 142, 331 (1998)

14. Dedner, A., F. Kemm, D. Kröner, C.-D. Munz, T. Schnitzer and M. Wesenberg: J. Comput. Phys., 175, 645 (2002)

15. H. De Sterck: 'Multi-Dimensional Upwind Constrained Transport on Unstructured Grids for 'Shallow Water' Magnetohydrodynamics'. In Proceedings of AIAA 15th Computational Dynamics Conference, (AIAA-2001-2623, 2001)

16. D. L. De Zeeuw and K. G. Powell: J. Comput. Phys., 104, 55 (1993)

17. D. L. De Zeeuw, S. Sazykin, A. J. Ridley, G. Tóth, T. I. Gombosi, K. G. Powell and R. A. Wolf: J. Geophys. Res., (2002) "in preparation",

18. Erickson, G. M., R. W. Spiro and R. A. Wolf: J. Geophys. Res., 96, 1633 (1991)

19. C. R. Evans and J. F. Hawley: Astrophys. Journ., 332, 659 (1988)

20. Fedder, J. A., S. P. Slinker, J. G. Lyon and R. D. Elphinstone: J. Geophys. Res., 100, 19,083 (1995) 
21. S. K. Godunov: 'Symmetric form of the equations of magnetohydrodynamics' (in Russian). In: Numerical Methods for Mechanics of Continuum Medium, "Siberian Branch of USSR Acad. of Sci.", 1, 26, Novosibirsk, 1972

22. T. I. Gombosi, D. L. De Zeeuw, R. M. Häberli and K. G. Powell: J. Geophys. Res., 101, 15233 (1996)

23. T. I. Gombosi, D. L. De Zeeuw, C. P. T. Groth, K. G. Powell and P. Song: The length of the magnetotail for northward IMF: Results of 3D MHD simulations, In Physics of Space Plasmas ed. by T. Chang and J. R. Jasperse, (MIT Press, Cambridge, Mass., 1998) pp. 121-128

24. T. I. Gombosi, D. L. De Zeeuw, C. P. T. Groth, K. G. Powell and Q. F. Stout: J. Atmos. Solar-Terr. Phys., 62, 1515 (2000)

25. T. I. Gombosi, G. Tóth, D. L. De Zeeuw, K. C. Hansen, K. Kabin and K. G. Powell: J. Comput. Phys., 177, 176 (2002)

26. C. P. T. Groth, D. L. De Zeeuw, T. I. Gombosi and K. G. Powell: J. Geophys. Res., 105, 25,053 (2000)

27. R. M. Häberli, T. I. Gombosi, D. L. DeZeuuw, M. R. Combi and K. G. Powell: Science, 276, 939 (1997)

28. K. C. Hansen, T. I. Gombosi, D. L. DeZeeuw, C. P. T. Groth and K. G. Powell: Adv. Space Res., 26, 1681 (2000)

29. Harel, M., R. A. Wolf, P. H. Reiff, R. W. Spiro, W. J. Burke, F. J. Rich and M. Smiddy: J. Geophys. Res., 86, 2217 (1981)

30. A. Harten: J. Comput. Phys., 49, 357 (1983)

31. R. K. Jaggi and R. A. Wolf: J. Geophys. Res., 78, 2842 (1973)

32. P. Janhunen: J. Comput. Phys., 160, 649 (2000)

33. K. Kabin, T. I. Gombosi, D. L. DeZeeuw, K. G. Powell and P. L. Israelevich: J. Geophys. Res., 104, 2451 (1999)

34. K. Kabin, T. I. Gombosi, D. L. De Zeeuw and K. G. Powell: "Icarus", 143, 397 (2000)

35. K. Kabin, M. R. Combi, T. I. Gombosi, D. L. De Zeeuw, K. C. Hansen and K. G. Powell: Planet. Space Sci., 49, 337 (2001)

36. R. Keppens, G. Tóth, M. A. Botchev and A. van der Ploeg: "Int. J. for Num. Meth. in Fluids", 30, 335 (1999)

37. P. D. Lax and B. Wendroff: Systems of Conservation Laws, "Communications on Pure and Applied Mathematics", 13, 217-237 (1960)

38. T. J. Linde, T. I. Gombosi, P. L Roe, K. G. Powell and D. L. De Zeeuw: J. Geophys. Res., 103, 1889 (1998)

39. T. J. Linde: A Three-Dimensional Adaptive Multifluid MHD Model of the Heliosphere", school = "Univ. of Mich.", Ann Arbor, month = may, 1998

40. Y. Liu, A. F. Nagy, C. P. T. Groth, D. L. De Zeeuw, T. I. Gombosi and K. G. Powell: Geophys. Res. Lett., 26, 2689 (1999)

41. B. Marder: J. Comput. Phys., 68, 48 (1987)

42. T. Ogino and R. J. Walker: Geophys. Res. Lett., 11, 1018 (1984)

43. H. Paillère, K. G. Powell and D. L. De Zeeuw: 'A wave-model based refinement criterion for adaptive-grid computation of compressible flows'. In: 30th AIAA Aerospace Sciences Meeting (AIAA-92-0322, Reno, Nevada, 1992)

44. K. G. Powell, P. L. Roe and J. Quirk: 'Adaptive-mesh algorithms for computational fluid dynamics'. In: Algorithmic Trends in Computational Fluid Dynmaics ed. by M. Y. Hussaini, A. Kumar and M. D. Salas (SPRINGER-VERLAG, New York, 1993) pp. 303-337 
45. K. G. Powell: 'An approximate Riemann solver for magnetohydrodynamics (that works in more than one dimension)'. Internal report at Inst. for Comput. Appl. in Sci. and Eng., NASA Langley Space Flight Center, Hampton, Va., 94-24, 1994

46. K. G. Powell, P. L. Roe, T. J. Linde, T. I. Gombosi and D. L. De Zeeuw: J. Comput. Phys., 154, 284 (1999)

47. J. J. Quirk: 'An Adaptive Grid Algorithm for Computational Shock Hydrodynamics'. Internal report at Cranfield Inst. of Technol., Cranfield, England", 1991

48. J. J. Quirk and U. R. Hanebutte: 'A parallel adaptive mesh refinement algorithm'. Interanl; report at ICASE, 93-63, 1993

49. J. Raeder, R. J. Walker and M. Ashour-Abdalla: Geophys. Res. Lett., 22, 349 (1995)

50. Richmond, A. D., E. C. Ridley and R. G. Roble: Geophys. Res. Lett., 19, 601 (1992)

51. Richmond, A. D.: J. Geomagn. Geoelectr., 47, 191 (1995)

52. A. J. Ridley, D. L. De Zeeuw, T. I. Gombosi and K. G. Powell: J. Geophys. Res., 106, 30,067 (2001)

53. A. J. Ridley, K. C. Hansen, G. Tóth, D. L. De Zueew, T. I. Gombosi and K. G. Powell: J. Geophys. Res., 107 (2002) "in press",

54. A. J. Ridley, T. I. Gombosi, D. L. De Zeeuw, C. R. Clauer and A. D. Richmond: J. Geophys. Res., 107 (2002) "in preparation"

55. A. J. Ridley, T. I. Gombosi and D. L. De Zeeuw: J. Geophys. Res. 107 (2002) "in preparation",

56. R.M. Robinson, R.R. Vondrak, K. Miller, T. Dabbs and D.A. Hardy: J. Geophys. Res., 92, 2565 (1987)

57. R. G. Roble and E. C. Ridley: Ann. Geophys., 5A, 369 (1987)

58. P. L. Roe: J. Comput. Phys., 43, 357 (1981)

59. P. L. Roe and D. S. Balsara: SOCIETY FOR INDUSTRIAL AND APPLIED MATHEMATICS J. APPL. MATH., 56, 57 (1996)

60. S. Sazykin and R. A. Wolf and R. W. Spiro and T. I. Gombosi and D. L. De Zeeuw and M. F. Thomsen: Geophys. Res. Lett., (2002) "in preparation"

61. P. Song, T.I. Gombosi, D.L. De Zeeuw, K.G. Powell and C. P. T. Groth: Planet. Space Sci., 48, 29 (2000)

62. P. Song, D. L. De Zeeuw, T. I. Gombosi, C. P. T. Groth and K. G. Powell: J. Geophys. Res., 104, 28,361 (1999)

63. J. M. Stone and M. L. Norman: Astrophys. J. Suppl., 80, 791 (1992)

64. T. Tanaka: J. Comput. Phys., 111, 381 (1994)

65. G. Tóth, R. Keppens and M. A. Botchev: Astron. Astrophys., 332, 1159 (1998)

66. G. Tóth: J. Comput. Phys., 161, 605 (2000)

67. G. Tóth and P. L. Roe: J. Comput. Phys., (2001) "submitted"

68. G. Tóth, D. L. De Zeeuw, T. I. Gombosi, K. C. Hansen, K. G. Powell, A. J. Ridley, P. L. Roe and I. V. Sokolov: J. Geophys. Res., 107 (2002) "in preparation"

69. B. van Leer: J. Comput. Phys., 32, 101, (1979)

70. V. M. Vasyliunas: 'Mathematical models of magnetospheric convection and its coupling to the ionosphere'. In: Particles and fields in the magnetosphere ed. by B. M. McCormack (D. Reidel Publishing, Dordrecht, Holland 1970) pp. 60-71

71. R. A. Wolf: 'The quasi-static (slow-flow) region of the magnetosphere'. In: Solar Terrestrial Physics, ed. by R. L. Carovillano and J. M. Forbes, (D. Reidel Publishing, Hingham, MA 1983) pp. 303-368 